\title{
Programação dinâmica aplicada à alocação de recursos no transporte de cargas
}

\author{
Antonio Martins Lima Filho; Nicolau D. Fares Gualda ${ }^{2}$
}

\begin{abstract}
Resumo: O planejamento operacional de um sistema de transporte de cargas de longa distância implica resolver um problema de otimização de rede dinâmica capacitada, visando a efetuar de forma eficaz e eficiente os movimentos das cargas, utilizando os recursos de transporte disponíveis. O presente trabalho aborda a utilização de programação dinâmica aproximada e adaptativa para solução desse tipo de problema. A metodologia de solução proposta substitui o processo de otimização global da rede por um modelo de Programação Dinâmica aplicado a cada estágio do problema; o método baseia-se em uma heurística para estimar o valor que a solução de cada estágio transfere para os estágios subseqüentes. A técnica utilizada reduz sensivelmente a quantidade de variáveis envolvidas, o que viabiliza a construção de modelos matemáticos mais realistas em um horizonte de planejamento mais amplo. São apresentados resultados de uma aplicação bem sucedida do modelo com utilização do Jensen Network Solver.
\end{abstract}

\begin{abstract}
Operational planning of a long haul transportation system implies to solve a capacitated dynamic network optimization problem, aiming to perform the freight movements in an efficient and effective way, utilizing the available transportation capacity. This work employs an approximate adaptive dynamic programming to solve this kind of problem. The proposed solution methodology replaces the network global optimization process by a dynamic programming model applied to each stage of the problem; the method is based on a heuristic to estimate the value that the solution of each stage transfers to further stages. This technique greatly reduces the quantity of involved variables, allowing the utilization of more realistic mathematical models in a longer planning horizon. Results from a successful application of the model with the utilization of Jensen Network Solver are presented.
\end{abstract}

\section{INTRODUÇÃO}

O problema considerado neste trabalho enquadra-se no conceito de Planejamento Operacional. De acordo com Gualda (1995), o planejamento operacional pressupõe a existência de um sistema físico implantado e visa à otimização operacional desse sistema. Powell (2003) identifica o Planejamento Operacional de Sistemas de Transporte com o Gerenciamento de Operações, em tempo real, e analisa, para cada tipo de serviço de transporte, os recursos que devem ser gerenciados e as decisões, as quais constituem a forma de gerenciamento desses recursos; para o autor, as decisões, por sua vez, devem levar em conta os processos a que se referem, ou seja, a dinâmica e as restrições do Sistema.

O Planejamento Operacional, para um sistema de transporte rodoviário de cargas em longa distância, é efetuado através do pessoal de gerenciamento local atuando em um ambiente altamente dinâmico, onde o fator tempo desempenha um importante papel e devem ser levados em conta os detalhes dos tipos de veículos, instalações e atividades. O planejamento pode ser definido como sendo uma ponte entre o conhecimento (dados, teoria) e a ação, ou tomada de decisão

\footnotetext{
${ }^{1}$ Antonio Martins Lima Filho, Escola Politécnica da Universidade de São Paulo. São Paulo, SP, Brasil. (e-mail: martins.lima@poli.usp.br).

${ }^{2}$ Nicolau D. Fares Gualda, Escola Politécnica da Universidade de São Paulo. São Paulo, SP, Brasil. (e-mail: ngualda@usp.br).
}

Manuscrito recebido em 14/7/2008 e aprovado para publicação em 15/10/2008. Este artigo é parte de TRANSPORTES, volume XVI, número 2, dezembro de 2008. ISSN: 1415-7713.
(Gualda, 1995).

No caso em questão, o Sistema é composto de cargas "full-truck-load" (uma única carga constituindo a lotação completa do veículo, a qual é transportada de uma única origem a um único destino). É considerado o caso em que a demanda pelo transporte ocorre em diversos pontos do espaço de forma aleatória, no decorrer do tempo. Uma característica básica deste tipo de transporte, definida através da expressão Longa Distância, é o fato de o veículo não retornar de imediato à base operacional de origem, depois de cumprida a entrega de sua carga. Dessa forma, para cada veículo tornado disponível, em cada ponto do espaço-tempo, deve-se tomar uma decisão de caráter operacional entre três possíveis alternativas: atender a uma carga nesse ponto, aguardar nesse ponto por uma carga futura, ou deslocar o veículo vazio para outro local.

No item 2 deste trabalho é apresentada uma revisão do problema na literatura. No item 3, a metodologia proposta para a sua solução. No item 4 é apresentada a solução de um problema típico. No item 5 são tecidas as conclusões do trabalho.

\section{ALOCAÇÃO DE RECURSOS NO TRANSPORTE DE CARGAS}

A necessidade de planejar a alocação de recursos é comum às várias operações de transporte, de cargas ou de passageiros, nos diversos modais. No caso do modal marítimo, contêineres vazios disponibilizados em uma determinada locação devem ser re-posicionados para outras locações, tendo em vista atender às demandas existentes ou previstas (White, 1972, Crainic; 
Gendrau; Dejax, 1993). Vagões ferroviários de carga também devem ser, em muitos casos, re-posicionados após a conclusão de uma tarefa; além disso, os vagões, vazios ou carregados, devem ser alocados a composições (Mendiratta; Turnquist, 1982, Jordan; Turnquist, 1983, Haghani, 1989). O problema de determinação do tipo de aeronave a designar para os diversos vôos programados por uma empresa de transporte aéreo é estudado por Hane et al. (1995). O modal aéreo apresenta ainda, como problema característico, a necessidade de alocação de tripulações às aeronaves, obedecendo a regras severas de regime de trabalho.

Para a alocação de veículos a cargas no transporte rodoviário de longa distância é necessário distinguir entre os serviços regulares, onde o planejamento tático é o mais importante, e os serviços não-regulares, onde o planejamento operacional se destaca. Para os serviços regulares podem ser citados os trabalhos de Crainic e Roy (1992) e de Powell e Sheffi (1989). Os serviços não-regulares, por sua vez, constituem o escopo do presente trabalho, bem como dos artigos, entre outros, de Frantzeskakis e Powell (1990), Godfrey e Powell (2002a, 2002b), Powell e Carvalho (1998), e Powell et al. (1995).

Os modelos de planejamento operacional de transporte podem ser estáticos ou dinâmicos. Os modelos estáticos se prestam melhor ao planejamento tático, onde o fator tempo pode ser deixado em segundo plano. Dessa forma, o problema de otimizar o transporte rodoviário de cargas em longa distância pode comportar uma abordagem estática e determinística, quando se tratar de planejamento em nível tático de serviços de transporte do tipo "não-regular". No caso do Transporte Rodoviário de Cargas em Longa Distância, a Alocação Dinâmica, mais que uma escolha de modelagem e de ferramenta de solução, é uma verdadeira necessidade imposta pela característica do problema: o recurso (equipamento de transporte), após servir uma tarefa, vai se encontrar disponível em uma localização diferente da original, onde pode ou não haver novas tarefas aguardando para serem servidas. A alocação de recursos aqui considerada é "dinâmica" em três diferentes sentidos (Powell; Jaillet; Odoni, 1995): o problema é dinâmico, uma vez que os dados mudam com o passar do tempo (os recursos são dinâmicos por se re-posicionarem ao término das tarefas); o modelo utilizado para representar o problema é também dinâmico, uma vez que incorpora explicitamente a interação de atividades através do tempo; e, finalmente, o método de solução (Programação Dinâmica) é constituído de repetidas soluções, à medida que nova informação se torna disponível. Um modelo dinâmico pode estar captando o estágio temporal das atividades físicas, mas não o estágio temporal da informação; neste caso o modelo é chamado determinístico. (Powell,
2003).

White (1972) formula o problema de distribuição de contêineres vazios como uma Rede de Transbordo Dinâmica. Os nós da rede correspondem a pontos no espaço e no tempo e às respectivas ofertas e demandas por contêineres, e os arcos representam os custos de deslocamento. A demanda é determinística e os tempos de partida dos deslocamentos são previamente fixados. O problema é solucionado através de uma versão do algoritmo out-of-kilter, proposto por Ford e Fulkerson (1962) para resolver problemas de circulação de custo mínimo. O algoritmo utilizado é indutivo, aproveitando a característica não cíclica da rede, ocasionada pela dimensão temporal na definição dos nós. $\mathrm{O}$ autor propõe ainda a utilização de técnicas de programação de característica dinâmica, visando a estender o horizonte de tempo: a cada vez que o modelo é resolvido, os dados são atualizados, eliminando-se o período inicial da iteração anterior e incorporando a informação relativa ao período imediatamente seguinte no horizonte de tempo. Haghani (1989) também propõe a utilização de uma rede espaço-tempo, incorporando características dinâmicas ao problema combinado de otimização da rede e formação de trens e da distribuição dos vagões vazios. A solução implica a utilização de uma técnica heurística de decomposição para o problema, o qual é formulado como programação "mixed-integer", com função objetivo não linear e restrições lineares.

Powell e Frantzeskakis (1994) introduzem a noção de "recurso nodal", substituindo o problema de transporte original por um problema de otimização mais simples, cuja solução produz uma boa aproximação da função de recurso. Uma função convexa de valor para os veículos disponíveis em determinado ponto do espaço tempo é submetida a um processo de linearização, de forma a se obter um valor médio, a partir do qual se constrói o algoritmo. Esta formulação dinâmica e estocástica mostrou-se superior a formulações alternativas, estáticas ou dinâmicas determinísticas, em experimentos conduzidos pelos autores através de uma técnica de horizonte rolante que possibilitou comparar os resultados obtidos em termos de frações do valor ideal (aquele que seria conseguido se a demanda futura fosse sempre conhecida).

Powell (1986) propõe representar o problema de alocação de recursos em transporte de longa distância na forma de uma rede espaço-tempo, como a da Figura 1, a seguir. Esta forma de modelagem produz uma rede de estrutura acíclica, facilitando a operação de algoritmos de otimização que tiram partido desta mesma estrutura. A dificuldade com este tipo de abordagem de rede é a grande extensão dos problemas envolvidos, em termos de quantidade de variáveis. Algumas poucas locações consideradas em um horizonte 
de tempo com alguns poucos períodos já produzem um problema de dimensão de difícil tratamento, especialmente quando se tenta a otimização pela via da programação linear inteira. Ademais, quaisquer detalhes da operação, como janelas de tempo, por exemplo, implicam novas multiplicações da quantidade de variáveis.

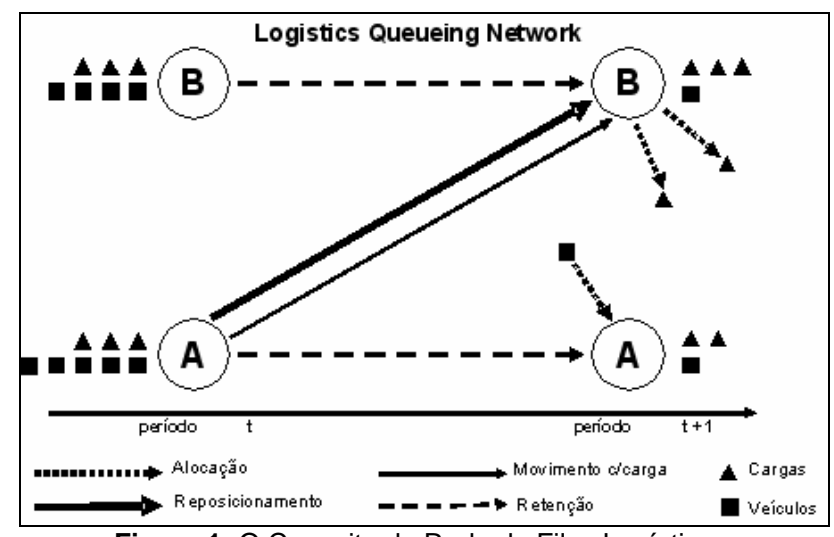

Figura 1: O Conceito de Rede de Filas Logísticas Fonte: Crainic (2003)

Uma forma de contornar as dificuldades de solução aqui apontadas é apresentada por Powell et al. (1995). Partindo da consideração dos chamados Sistemas Dinâmicos de Eventos Discretos (Discrete Event Dynamic System - DEDS), os autores propõem uma nova abordagem, onde demandas são enfileiradas em terminais enquanto aguardam pela disponibilização de capacidade de atendimento e, da mesma forma, os equipamentos ao serem disponibilizados se enfileiram aguardando designação. O modelo, assim formulado, assume a característica de uma rede com filas duplas (cargas e equipamentos) em cada um dos nós. Daí a denominação "Rede de Filas Logísticas" (Logistics Queueing Network - LQN). A Figura 1, com base em Crainic (2003), constitui uma representação deste modelo.

A proposta, então, é substituir a solução global de otimização através de Programação Linear por uma solução de Programação Dinâmica Recursiva, permitindo decompor a solução geral do problema de rede em uma série de soluções de subproblemas em nível de cada uma das locações. A abordagem geral de solução consiste de uma série de passos para frente e para trás, através da linha do tempo: a cada iteração, o passo para a frente designa veículos a cargas e efetua, de forma limitada, re-posicionamentos de veículos; o passo para trás calcula gradientes, e uma fase de ajuste de controles modifica os valores potenciais e os limites dos movimentos em vazio. O processo continua até que se assegura um nível desejado de convergência (Crainic, 2003).

Powell et al. (1995) e Powell e Carvalho (1998) propõem, com base nesse modelo, soluções que se aplicam a problemas determinísticos, limitação supera- da a partir do desenvolvimento do algoritmo CAVE (Concave Adaptive Value Estimation) de Godfrey e Powell (2001), capaz de efetuar uma estimativa acurada de funções côncavas de valor com base nos resultados de uma sucessão de experiências, sem depender de se conhecer a específica função de probabilidades associada aos eventos. O algoritmo se baseia na geração de gradientes para a quantidade de recursos disponibilizada, levando em conta o resultado obtido em vista da demanda realmente ocorrida. A partir daí, o algoritmo efetua uma alteração suavizada na forma da curva para utilização na iteração seguinte. Essa alteração de forma da curva é efetuada em intervalos de tempo de magnitude pré-estabelecida em torno do ponto considerado, e se faz de maneira a preservar sempre a concavidade da função.

Godfrey e Powell (2002a e 2002b) efetuam uma modelagem e propõem uma solução centrada basicamente em duas reduções de complexidade: o problema é separado por locação / período (conceito de Rede de Filas Logísticas, conforme descrito acima) e depois é resolvido utilizando uma estimativa do valor associado aos estados futuros (o algoritmo CAVE, descrito anteriormente). A essência do modelo é apresentada a seguir.

\section{METODOLOGIA}

A metodologia adotada neste trabalho baseia-se na utilização de Programação Dinâmica, Estocástica, Aproximada e Adaptativa, aplicada ao Planejamento Operacional de um Sistema de Transporte Rodoviário de Cargas em Longa Distância, tendo em vista maximizar o resultado econômico do Sistema, em função da alocação de equipamentos de transporte a cargas. A técnica utilizada baseia-se no modelo proposto por Godfrey e Powell (2002a e 2002b) para a otimização da alocação de recursos (frotas de veículos) a tarefas (cargas disponíveis para transporte), em diferentes pontos no espaço e no tempo; após o atendimento, o recurso se torna disponível e pode então ser utilizado na mesma locação (imediatamente ou após algum tempo de espera) ou ser re-posicionado para outra locação. Nesses trabalhos é utilizado o conceito de Rede de Filas Logísticas (Powell et al., 1995, Powell; Carvalho, 1998), o qual permite resolver subproblemas correspondentes a períodos de tempo individualizados, evitando a necessidade de tratar a rede como um todo. O modelo utiliza, ainda, uma técnica de estimativa de valores de funções côncavas proposta por Godfrey e Powell (2001) denominada CAVE (Concave Adaptive Value Estimation), para definir os valores correspondentes, em cada subproblema, à melhor solução para o restante do período de planejamento. 


\subsection{Modelo de programação dinâmica estocástica}

Sejam:

$T$ : número de períodos no horizonte de planejamento;

$\tau: \quad\{0,1, \ldots ., T-1\}=$ tempos em que as decisões são tomadas;

J : conjunto de locações físicas na rede, indexadas por $i$ e $j$;

$\mathcal{L}^{+}{ }_{i t}$ : conjunto das cargas a serem atendidas no tempo t a partir da locação $i$;

$\mathbb{R}_{i t}^{+}$: quantidade total de recursos na locação $i$ disponíveis para atuar no tempo $t$;

As variáveis de decisão, para cada $t \in \mathcal{T} ; i, j \in \mathcal{J}$, $l \in \mathcal{L}^{+}{ }_{i t}$, podem ser representadas por:

$$
x_{l t}= \begin{cases}1 \quad \begin{array}{l}
\text { se um recurso em } i \text { é designado } \\
\text { para uma tarefa } l \in \mathcal{L}^{+}{ }_{i t} \text { no tempo } t
\end{array} \\
0 \quad \text { caso contrário } \\
y_{i j t}=\quad \begin{array}{l}
\text { quantidade de recursos reposicio- } \\
\text { nados de } i \text { para } j \text { iniciando-se no } \\
\text { tempo } t
\end{array}\end{cases}
$$

Inicialmente, para um particular período de tempo $t$, pode ser definida a seguinte função de recompensa:

$$
g_{t}\left(x_{t} y_{t}\right)=\sum_{i \in \mathcal{J}} \sum_{l \in \mathcal{L}_{i t}^{+}} r_{l t} x_{l t}-\sum_{i \in \mathcal{J}} \sum_{j \in \mathcal{J}} c_{i j} y_{i j t}
$$

em que,

$r_{l t}:$ "recompensa" obtida por atender a tarefa $l \in \mathcal{L}^{+}{ }_{i t}$ iniciando-se no tempo $t \in \mathcal{T}$;

$c_{i j}$ : "custo" de reposicionar um recurso da locação $i$ para a locação $j$.

Considerando agora todo o horizonte de planejamento, a função objetivo, em termos de Programação Dinâmica Estocástica, é dada por:

$$
\max _{x_{0}, y_{0} \in \mathcal{H}_{0}} g_{0}\left(x_{0} y_{0}\right)+E\left\{\sum_{t \in \mathcal{T} \backslash 0} \max _{x_{t}, y_{t} \in \mathcal{H}_{t}} g_{t}\left(x_{t} y_{t}\right)\right\}
$$

em que $\mathcal{H}_{\mathrm{t}}$ é o $\sigma$-álgebra (subconjunto de eventos do espaço amostral $\Omega$ para o qual a medida de probabilidade $\mathrm{P}$ é definida) gerado por $\left(W_{\mathrm{o}}, \ldots, W_{\mathrm{t}}\right)$, sendo $W_{t}=\left(\hat{R}_{t}, \hat{\mathcal{L}}_{t}\right)$ a nova informação (recursos disponíveis, tarefas a atender) chegando no período de tempo $t$.

As restrições são de 2 tipos, aplicando-se às decisões e à dinâmica do sistema:

$1^{\circ}$. restrições que se aplicam às decisões tomadas em um ponto no tempo $t$ sob realização estocástica $\omega \in \Omega$ :

$$
\sum_{j \in \mathcal{J}} y_{i j t}(\omega)+\sum_{l \in \mathcal{L}_{i t}^{+}(\omega)} x_{l t}(\omega)=R_{i t t}^{+}(\omega) \quad \forall i \in \mathcal{J}
$$

$$
\begin{array}{cc}
x_{l t}(\omega) \leq 1 & \forall\left[\in \mathcal{L}_{t}^{+}(\omega)\right. \\
x_{l t}(\omega) \geq 0 & \forall\left[\in \mathcal{L}_{t}^{+}(\omega)\right. \\
y_{i j t}(\omega) \geq 0 & \forall i, j \in \mathcal{J}
\end{array}
$$

$2^{\circ}$. - restrições que controlam a dinâmica do sistema no tempo:

$R_{j, t+1, t+\tau}(\omega)=\sum_{i \in \mathcal{J}: \tau_{i j}=\tau}\left(y_{i j t}(\omega)+\sum_{l \in \mathcal{L}_{i j t}^{+}(\omega)} x_{l t}(\omega)\right)+R_{j, t, t+\tau} \forall j \in \mathcal{J}$

em que:

$\tau_{i j}: \quad$ o tempo de viagem de $i$ a $j ; i, j \in \mathcal{J}$

$$
\mathcal{L}_{t+1}(\omega)=\mathcal{L}_{t}^{+}(\omega) \backslash \mathcal{L}_{t}^{e}(\omega) \forall\left(\tau=1, \ldots ., \tau_{\max }\right)
$$

sendo:

$\tau_{\max }:$ o maior tempo de viagem entre quaisquer pares de locações, e

$\mathcal{L}_{t}^{e}$ : conjunto das tarefas que expiram no tempo $t$ (porque foram atendidas ou porque deixaram o sistema devido a alguma outra razão)

\subsection{Estratégia de solução por programa dinâmica adaptativa}

Em aplicações práticas para esta classe de problemas, a estratégia de solução mais natural é utilizar um procedimento de horizonte rolante, resolvendo o problema no tempo $t$, utilizando o que é conhecido no tempo $t$ e mais uma previsão de eventos futuros sobre algum horizonte de tempo (Godfrey; Powell, 2002a e 2002b). Para tanto, faz-se necessário definir uma função de valor para o período $t$, com base em uma estimativa de função de valor para o período $t+1$ :

$$
\tilde{V}_{t}\left(R_{t}, \omega_{t}\right)=\max _{x_{t}\left(\omega_{t}\right), y_{t}\left(\omega_{t}\right) \in \mathcal{H}_{t}\left(R_{t}, \omega_{t}\right)} g_{t}\left(x_{t}\left(\omega_{t}\right), y_{t}(\omega t)\right)+\hat{V}_{t+1}\left(R_{t+1}\left(\omega_{t}\right)\right)
$$

Godfrey e Powell (2002a) utilizam uma estimativa de Função de Valor separada por locação, na forma $\hat{V}_{t}\left(R_{t}\right)=\Sigma \hat{V}_{i t}\left(R_{i t}\right)$, onde as funções $\hat{V}_{i t}\left(R_{i t}\right)$ são estimadas utilizando o algoritmo CAVE (Godfrey; Powell, 2001). O problema é resolvido executando-se um "passo para a frente" no tempo, determinando um conjunto de decisões $\left(x_{t}\right)_{t} \in \mathcal{T}$, para uma particular amostra $\omega$, e usando um particular conjunto de aproximações da função de valor.

Ao final do "passo para a frente", as variáveis duais obtidas da solução de cada subproblema são utilizadas para atualizar as aproximações funcionais usando o algoritmo CAVE (Godfrey; Powell, 2001). A solução do problema é encontrada após sucessivas aproximações das variáveis de decisão ("passos para a frente"), seguidas das respectivas atualizações das 
funções de valor. Ou seja, conhecida a estimativa de função de valor e um resultado aleatório $\omega \in \Omega$ para as demandas futuras, resolve-se a seguinte seqüência de subproblemas de rede, começando com $t=0$ e continuando até $t=T-1$ :

$$
\max _{x_{t}, y_{t} \in \mathcal{H}_{t}} g_{t}\left(x_{t} y_{t}\right)+\sum_{\tau=1}^{\tau_{\max }} \hat{V}_{t+1, t+\tau}\left(R_{t+1, t+\tau}\right)
$$

O subproblema (10) no tempo $t$ pode ser visto como uma rede de 2 estágios, como ilustrado na Figura 2.

Figura 2: Rede de 2 estágios correspondente ao subproblema

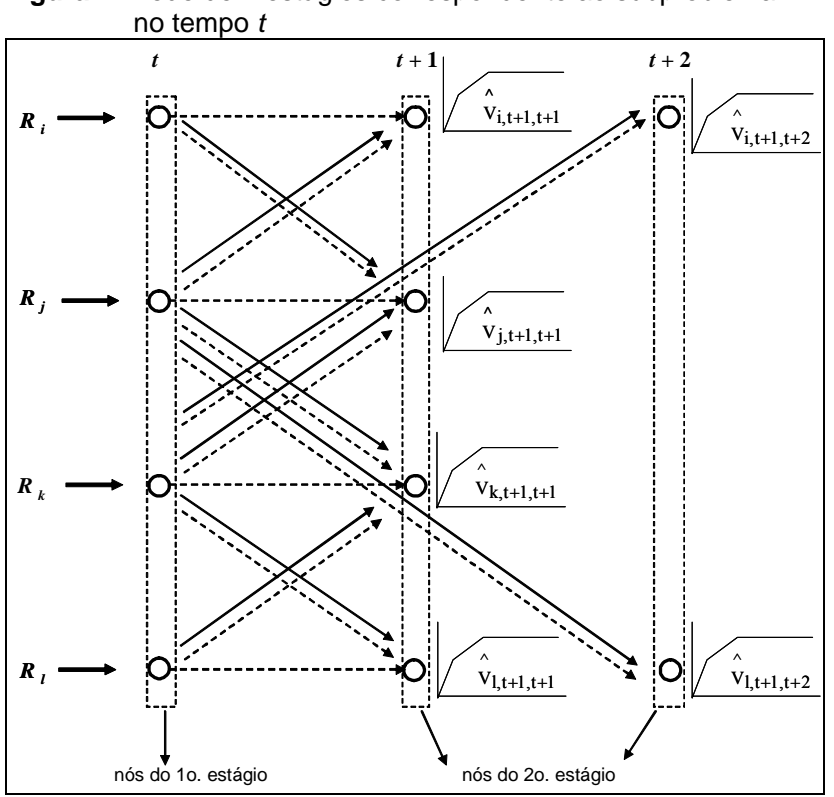

Para o $1^{\circ}$ estágio, as setas em linha cheia indicam movimentos com carga, os quais têm "custo" (recompensa) $=r_{l t}$ e limite superior $\mathcal{L}^{+}{ }_{i t}(\omega)$; as setas em linha pontilhada representam reposicionamentos de veículos sem carga, com custo $=-c_{i j}$ e limite superior $=+\infty$. As decisões de $2^{\circ}$ estágio (tempos $t^{\prime}=t+\tau, \tau>0$ ) incorporam a aproximação de Função de Valor $\hat{V}_{t+1, t^{\prime}}\left(R_{t+1, t^{\prime}}\right)$.

\section{TESTE DO MODELO}

Tendo em vista assegurar o entendimento do modelo e a eficácia de seus resultados, foi desenvolvido um experimento utilizando um problema dimensionado de forma a permitir a sua otimização global, através de um software comercial comum, além da análise do comportamento do sistema de forma visual. Este problema consiste em 3 locações e horizonte de planejamento de 3 períodos, alocando um total de 6 veículos.

Com a finalidade de simplificar o modelo, mas sem prejuízo da avaliação de sua eficácia, os tempos de trânsito foram considerados como unitários e a disponibilidade de cargas a atender foi considerada de forma determinística. Pode-se afirmar que a extensão do experimento para problemas multiperíodo e probabi- lísticos estará garantida, uma vez que, para tanto, é suficiente alterar as características da rede e a forma de atribuir quantidades de demanda de uma para a próxima iteração.

O problema pode ser representado como uma rede de circulação de receita máxima, consistindo de 13 nós (incluindo o sorvedouro) e 49 arcos. A solução de otimização foi obtida através do Jensen Network Solver (Jensen; 2008), para a rede global, utilizando um modelo linear e inteiro. Foram simuladas 20 diferentes configurações de valor para a mesma estrutura básica de rede, variando-se ora o limite superior de algum arco (quantidade de cargas a atender), ora o valor da recompensa por atender a alguma carga (custo do arco), ora a distribuição do veículo entre as 3 locações no período inicial.

Foi aplicado, a seguir, para as mesmas 20 configurações, o modelo de solução através da divisão do problema em 3 subproblemas, um para cada período. Cada subproblema foi configurado como uma rede de circulação com 7 nós e 34 arcos e a solução foi obtida com a utilização do mesmo software, na mesma estrutura de modelo linear e inteiro. As iterações foram efetuadas até que a melhora no valor do resultado ótimo (considerando o $1^{\circ}$ e o $2^{\circ}$ estágio da rede) em relação à iteração anterior fosse inferior a $0,5 \%$. A quantidade de iterações variou, de acordo com a configuração adotada, de um mínimo de 10 até um máximo de 16. O resultado ótimo foi obtido já na $2^{\mathrm{a}}$ iteração, para o caso mais favorável, e na $7^{\mathrm{a}}$ iteração para o caso mais complexo.

Neste experimento foi adotado um fator de suavização de 0,5 para todas as iterações, ou seja, a função de valor foi atualizada, de uma iteração para a seguinte, pela média entre o acumulado até a iteração e o valor da nova iteração considerada. Este fator deve ser calibrado para o particular caso tratado, tendo-se em conta que valores mais elevados estarão conduzindo a uma solução mais rápida, porém em detrimento da conservação da memória dos valores anteriormente considerados, o que compromete a estimativa da função densidade de probabilidade, em problemas estocásticos.

O valor obtido utilizando-se a técnica de subproblemas, em todos os experimentos, foi idêntico ao valor ótimo fornecido pelo processo de otimização global da rede, o que comprova a eficácia do modelo quando aplicado com a técnica utilizada no experimento.

A configuração do experimento em que a otimização foi obtida apenas na $7^{\mathrm{a}}$ iteração é de particular interesse para a análise do funcionamento do modelo. Neste caso, a demanda para cargas na locação 3 no terceiro período de tempo (5 cargas) apresentou uma quantidade sensivelmente superior às demais quantidades demandas e, além disso, um valor de recompen- 
sa pelo atendimento fortemente mais atrativo que os demais. Tendo em vista a disponibilidade total de veículos ser de apenas 6 , a cada iteração a função de valor nesse ponto (locação $3,3^{\circ}$ período) apresentou um incremento suficiente para propiciar o direcionamento da rede em função dessas cargas privilegiadas em recompensa. O gráfico apresentado na Figura 3 mostra a evolução da função de valor (as linhas correspondem às 16 iterações, da inferior para a superior). Pode-se notar a convergência de valores à medida que as iterações ocorrem; as últimas 6 funções aparecem, no gráfico, como praticamente uma só linha.

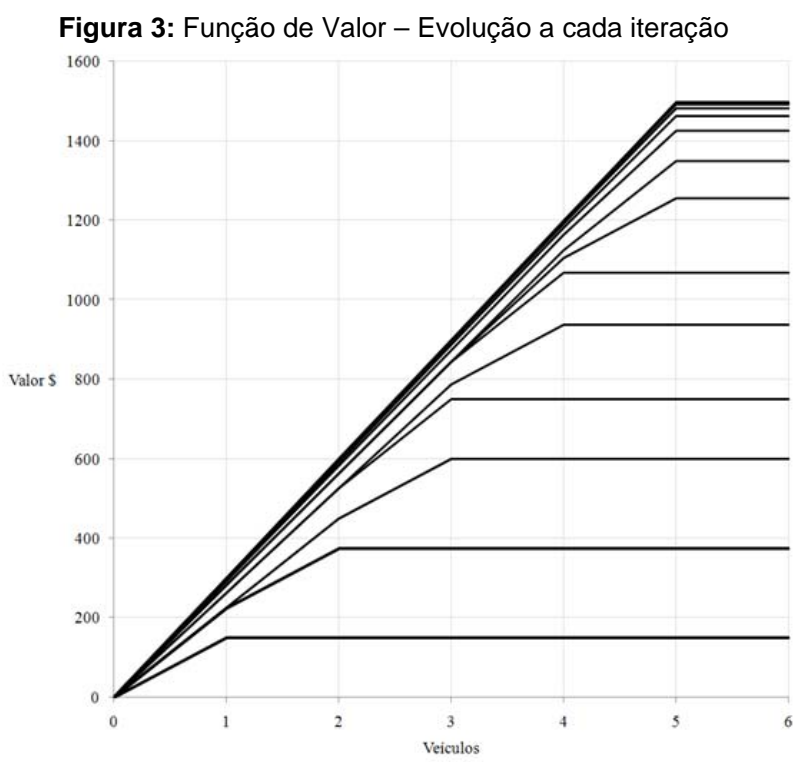

Embora o problema teste tenha dimensões relativamente reduzidas, o tempo de processamento computacional foi da ordem de 1 segundo, o que permite considerar a sua aplicabilidade a casos de dimensões sensivelmente maiores. Cabe lembrar que a solução em estágios, própria da Programação Dinâmica, não implica aumento do tamanho do problema a resolver em cada estágio se o horizonte de tempo a considerar for estendido. Passa a haver, simplesmente, uma maior quantidade de subproblemas (estágios) a computar. Assim, se o problema resolvido tivesse um horizonte de 30 períodos, a solução do problema de otimização global consumiria um tempo de computação maior que a solução para 3 períodos, embora o tempo de solução para cada subproblema permanecesse o mesmo.

\section{CONCLUSÕES}

Neste trabalho foi apresentado um método de Programação Dinâmica Adaptativa para a solução do problema de alocação de recursos de transporte a cargas rodoviárias de longa distância. $\mathrm{O}$ método consiste na resolução de subproblemas correspondentes a cada período de tempo, utilizando-se uma estimativa de função de valor para a otimização da rede em todo o horizonte de planejamento.
Os testes efetuados mostram que o tempo de processamento para os subproblemas independe da magnitude do horizonte de planejamento, enquanto o tempo de processamento para a solução do problema global cresce à medida que se consideram problemas de maior dimensão. Estes resultados permitem que o método seja estendido para problemas de dimensões reais; neste momento, está sendo desenvolvida uma aplicação do método para o transporte rodoviário de cargas no Mercosul, utilizando um modelo estocástico e multiperíodo.

Considera-se, ainda, a possibilidade de uma linha futura de pesquisa para tratar o caso de veículos heterogêneos (Topaloglu; Powell, 2006). O método apresentado, por outro lado, pode ser aplicado à solução de problemas de Programação Dinâmica em geral, o que viabiliza a sua utilização para sistemas de apoio à decisão associados a atividades cujas características conduzem naturalmente a essa forma de modelagem.

\section{AGRADECIMENTOS}

Os autores agradecem o suporte técnico do LPT / EPUSP - Laboratório de Planejamento e Operação de Transportes da Escola Politécnica da Universidade de São Paulo.

\section{REFERÊNCIAS BIBLIOGRÁFICAS}

Crainic, T.G. (2003) Long-haul freight transportation. In: Handbook of transportation science, Randolph W. Hall (ed.). 2a. edição. Boston: Kluwer Academic Publishers.

Crainic, T., Gendreau, M. e Dejax, P. (1993) Dynamic stochastic models for the allocation of empty containers. Operations Research, v. 41, p. 102-126.

Crainic, T., Roy J. (1992) Design of regular intercity driver routes for the LTL motor carrier industry. Transportation Science, Baltimore, v. 26, p. 280-295.

Ford, L.R., Fulkerson, D.R. (1962) Flows in Networks. New Jersey: Princeton University Press.

Frantzeskakis, L.F., Powell, W.B. (1990) A successive linear approximation procedure for stochastic, dynamic vehicle allocation problems. Transportation Science, Baltimore, v. 24 (1), p. 40-57.

Godfrey, G.A.; Powell, W.B. (2001) An adaptive, distribution free algorithm for the Newsvendor Problem with censored demands, with applications to inventory and distribution problems. Management Science, v. 47 (8).

Godfrey, G.A., Powell, W.B. (2002a) An adaptive dynamic programming algorith for dynamic fleet management, I: single period travel times. Transportation Science, Baltimore, v. 36 (1), p. 21-39.

Godfrey, G.A., Powell, W.B. (2002b) An adaptive dynamic programming algorithm for dynamic fleet management, II: multiperiod travel times. Transportation Science, Baltimore, v. 36 (1) p. 40-54.

Gualda, Nicolau Dionísio Fares (1995) Terminais de transportes: contribuição ao planejamento e ao dimensionamento operacional. Tese (Livre-Docência) - Departamento de Engenharia de Transportes, Escola Politécnica da Universidade de São Paulo, São Paulo.

Haghani, A. (1989) Formulation and solution of a combined train routing and makeup, and empty car distribution model. Transportation Research, v. 23B (6), p. 433-452, 1989

Hane, C.; Barnhart, C.; Johnson, E.; Marsten, R.; Nemhauser, G.; Sigismondi G. (1995) The fleet assignment problem: solving a largescale integer program. Mathematical Programming, Amsterdan, v. 70, p. 211-232.

Jensen, P.A. (2008) Operations Research Models and Methods. Internet: http://www.me.utexas.edu/ jensen/ORMM/. Acesso em $13 \mathrm{fev.}$ 2008

Jordan, W., Turnquist, M. (1983) A stochastic dynamic network model for railroad car distribution. Transportation Science, Baltimore, v. 17, p. 123-145. 
Mendiratta, V., Turnquist, M. (1982) A model for the management of empty freight cars. Transportation Research Record, v. 838, p. $50-55$.

Powell, W.B. (1986) A stochastic model of the dynamic vehicle allocation problem. Transportation Science, v. 20 (2), p. 117-129.

Powell, W.B. (2003) Dynamic models of transportation operations. In: Handbooks in Operations Research and management science, 11: Supply Chain Management: design, coordination and operation. A.G. de Kok e Stephen C. Graves (eds), Amsterdan; Boston: Elsevier.

Powell, W.B.; Carvalho, T.A. (1998) Dynamic control of logistics queueing networks for large-scale fleet management. Transportation Science, v. 32 (2), p. 90-109.

Powell, W.B.; Carvalho, T.A.; Godfrey, G.A.; Simão. H.P. (1995) Dynamic fleet management as a logistics queueing network. Annals of Operations Research, v. 61, p. 165-168.

Powell, W.B.; Frantzeskakis, L. (1994) Restricted recourse strategies for dynamic networks with random arc capacities. Transportation Science, v. 28 (1), p. 3-23.

Powell, W.B.; Jaillet, P.; Odoni, A. (1995) Stochastic and dynamic networks and routing. In: Handbook in Operations Research and Management Science, 8: Network Routing. M.O. Ball et al. (eds), Amsterdan; Boston: Elsevier, p. 141-295.

Powell, W.B., Sheffi, Y. (1989) Desgin and implementation of an interactive optimization system for network design in the motor carrier industry. Operations Research, v. 37 (1), p. 12-29.

Topaloglu, H. ; Powell, W.B. (2006) Dynamic programming approximations for stochastic time-staged integer multicommodity-flow problems. INFORMS Journal on Computing, v. 18 (1), p. 31-42.

White, W.W. (1972) Dynamic transshipment networks: an algorithm and its application to the distribution of empty containers, Networks, v. 2, p. 211-236. 